\title{
THE EFFECT OF VOXEL SIZE ON THE RELIABILITY AND REPRODUCIBILITY OF ALVEOLAR BONE CREST IDENTIFICATION ON CONE BEAM CT SCANS
}

\author{
Nora Saif* and Amr Ragab El-Beialy**
}

\begin{abstract}
Objective: the aim of this study is to compare the reliability and reproducibility of landmarks representing the labial alveolar bone crest of maxillary and mandibular teeth using two different CBCT machines with different image resolutions and voxel size and accordingly different radiation dose.

Material \& Methods: 340 landmarks representing the alveolar bone crest opposite each tooth from the central incisor up to the second molar on sixteen CBCT data sets were identified. The landmarks were located by the principle operator, and relocated by the same principle operator to evaluate the intra-observer reproducibility, then located by the second observer to evaluate the interobserver reliability error. For testing the intra-observer reproducibility and inter-observer reliability of the landmark identification within each scanner data, the Mean Absolute error (MAE) and the Dahlberg error (DE) and Intra-class correlation coefficient tests with $95 \%$ confidence intervals were used. Independent samples t-test was used to compare the intra-observer and inter-observer error between the two scanners.
\end{abstract}

Results: The Mean Absolute Error (MAE), the Dahlberg Error (DE) and the Intra-Class Coefficient (ICC) tests showed excellent intra-observer reproducibility values in the $\mathrm{x}, \mathrm{y}$ and $\mathrm{z}$-axis for the Galileos scanner, with the least reproducibility in the z-axis. The same concordance was found for the Planmeca CBCT scanner, with excellent reliability in the $\mathrm{x}, \mathrm{y}$ and $\mathrm{z}$-axis, and the least reproducibility in the z-axis. For the inter-observer reliability testing, the same pattern was found for each CBCT scanner. Very good inter-observer reliability was found in the $\mathrm{x}$, and y dimensions, while the least was found in the vertical z-dimension.

Conclusion: The Galileos and the Planmeca CBCT scanners produced comparable reliability and reproducibility for identification of alveolar bone crest landmarks. The vertical axes showed the least accurate coordinates for landmarks identification.

* Lecturer, Oral and Maxillofacial Radiology, Faculty of Dentistry, Cairo University, Egypt

** Lecturer, Department of Orthodontics, Faculty of Dentistry, Cairo University, Egypt 


\section{INTRODUCTION}

The perpetual alliance between the teeth and the embracing alveolar bone renders the evaluation of the alveolar bone an everlasting research point each time a new diagnostic tool is invented. Currently, although the pendulum of dentofacial diagnosis is swinging towards $3 \mathrm{D} \mathrm{CBCT}$, ${ }^{[1]}$ the argument between the proponents of the ALARA (As Low As Reasonably Achievable) philosophy and the eagerness of the maxillofacial community for this new technology is not settled so far. Concerning the use of CBCT to analyze the alveolar bone, some evidence should be considered.

Firstly, there is a consensus of evidence that a mutual relationship exists between the teeth and the supporting alveolar bone. The presence of buccal alveolar bone defects decreases the bony support for the teeth. ${ }^{[2]}$ The lack of bony support during orthodontic movement can be detrimental to the health of the periodontium and consequently the teeth,,$^{[2-5]}$ a potential factor for orthodontic relapse, ${ }^{[6-8]}$ and gingival recession. ${ }^{[4,9-11]}$ On the other hand orthodontic tooth movement can create alveolar bone defects. ${ }^{[12-14]}$ The correlation between the labial tipping of teeth and the associated changes in the labial alveolar plate is factual in some studies. $[11,15,16]$ Posteriorly, the $5 \mathrm{~mm}$ of buccal bone loss following rapid palatal expansion is striking. ${ }^{[17]}$

The capability to accurately evaluate the alveolar bone defects 3-dimensionally via CBCT is a leap in orthodontic diagnosis ${ }^{[18-22]}$ with a direct impact on the treatment decision.

The main advantage of CBCT is its lower acquisition time and patient dose when compared to medical CT scanning ${ }^{[23-26]}$. However, CBCT devices and different imaging protocols should not be addressed as a single entity. The image quality of CBCT scans and accordingly the ability of CBCT to display anatomic features or pathology depends on acquisition parameters such as milliamperage, kilovoltage and also spatial resolution defined by the voxel size ${ }^{[27]}$. The ability to view periodontal structures such as the periodontal ligament space, cortical bone, alveolar crest and alveolar cortical plate requires images with a higher definition and smaller voxel size, resulting in increased milliamperage and kilovoltage values. ${ }^{[28-30]}$

Images acquired in smaller voxel sizes will increase the radiation dose to the patient but might provide the same diagnostic outcome as lower resolution image ${ }^{[31]}$. Thus, it is important to consider that the comparison of CBCT examinations with various voxel settings is relevant to understand the impact of the inherent image quality on the reliability and reproducibility of a specific diagnostic outcome ${ }^{[21]}$.

Accordingly, the aim of this study is to compare the reproducibility and reliability of landmarks representing the labial alveolar bone crest of maxillary and mandibular teeth using two different CBCT machines with a different image resolution and accordingly a different radiation dose.

\section{MATERIAL AND METHODS}

In the current study, Cone Beam CT volumes were utilized to evaluate the accuracy of identification of the alveolar bone crest landmarks on CBCT data from two different CBCT scanners. The inclusion criteria for enrollment of the patient CBCT in this study is the presence of erupted teeth, and good quality CBCT data with absence of motion of the patient during the image capturing procedure. The scanners used were the Galileos* and the Planmeca Promax3D Mid** CBCT scanners. The parameters used during imaging using the Galileos scanner (CBCT scanner A) and the Planmeca scanner (CBCT scanner B) are shown in Table 1.

* Sirona Dental Systems GmbH-Operating Instructions GALILEOS

** ProMax 3D by Planmeca Oy, Helsinki, Finland-Operating Instructions ProMax 3D ${ }$ 
TABLE (I) Technical parameters and settings of the Galileos and ProMax 3D CBCT scanners*

\begin{tabular}{|c|c|c|}
\hline Technical Parameter & Galileos Scanner & ProMax 3D Scanner \\
\hline X-ray source voltage & $85 \mathrm{kVp}$ & $90 \mathrm{kVp}$ \\
\hline X-ray source current & $7 \mathrm{~mA}$ & $8 \mathrm{~mA}$ \\
\hline Scanning time & Approx. $14 \mathrm{~s}$ & $13.542 \mathrm{~s}$ \\
\hline Radiation Source & Pulsed & Pulsed \\
\hline Detector type & Image Intensifier-CCD & Flat panel \\
\hline Voxel size $(\mathrm{mm})$ & $0.3 \times 0.3 \times 0.3 \mathrm{~mm}^{3}$ & $0.4 \times 0.4 \times 0.4 \mathrm{~mm}^{3}$ \\
\hline Scanned volume dimensions (Field of view) & $15 \mathrm{~cm} \times 15 \mathrm{~cm} \times 15 \mathrm{~cm}$ & $20.0 \times 20.0 \times 20.0 \mathrm{~cm}$ \\
\hline Patient positioning & $\begin{array}{l}\text { Standing or seated with flat occlusal } \\
\text { plane }\end{array}$ & $\begin{array}{l}\text { Standing or seated with flat occlusal } \\
\text { plane }\end{array}$ \\
\hline Data output & DICOM & DICOM \\
\hline Effective dose value $\left(D_{\text {eff }}\right)$ & ICRP 2007=54 mSv* & $23 \mathrm{mSv} \neq$ \\
\hline Focal spot size & $0.5 \mathrm{~mm}$ & $0.5 \mathrm{~mm}$, fixed anode \\
\hline Primary reconstruction time & Approx. 2.5 minutes & $55 \mathrm{~s}$ \\
\hline
\end{tabular}

* “Comparative dosimetry of GALILEOS Dental CBCT imaging,” Prof. Dr. John B. Ludlow, Department of Diagnostic Sciences and General Dentistry, University of North Carolina School of Dentistry, Chapel Hill, North Carolina, USA

\$ Rottke, D et al. "Effective Dose Span of Ten Different Cone Beam CT Devices." Dentomaxillofacial Radiology 42.7 (2013): 20120417. PMC. Web. 5 Feb. 2018

Three hundred and forty landmarks representing the alveolar bone crest opposite each tooth from the central incisor up to the second molar on sixteen CBCT data were identified on each CBCT volume. The orthogonal cuts were used to accurately identify the location of the alveolar bone crest landmarks at the depth of the alveolar bone in the midline section of each tooth (Fig.1) The x, y, and z-coordinates for each landmark were recorded by the primary observer. For intra-observer reliability testing, the same landmarks were re-identified by the same observer 2 weeks after the first observation. The second observer identified the same landmarks and recorded the $\mathrm{x}, \mathrm{y}$, and $\mathrm{z}$-coordinates of each landmark.

Statistical analysis was performed using SPPS/ SPSS ${ }^{\circledR}$ v. 17 (IBM corporation, Armonk, NY). For testing the intra-observer reliability of the landmark identification within each scanner data, the Mean Absolute error (MAE) and the Dahlberg error (DE) and Intra-class Correlation coefficient tests with 95\% confidence intervals were used. Independent samples t-test was used to compare the intraobserver error between the two scanners, as well as the inter-observer error between the two scanners.

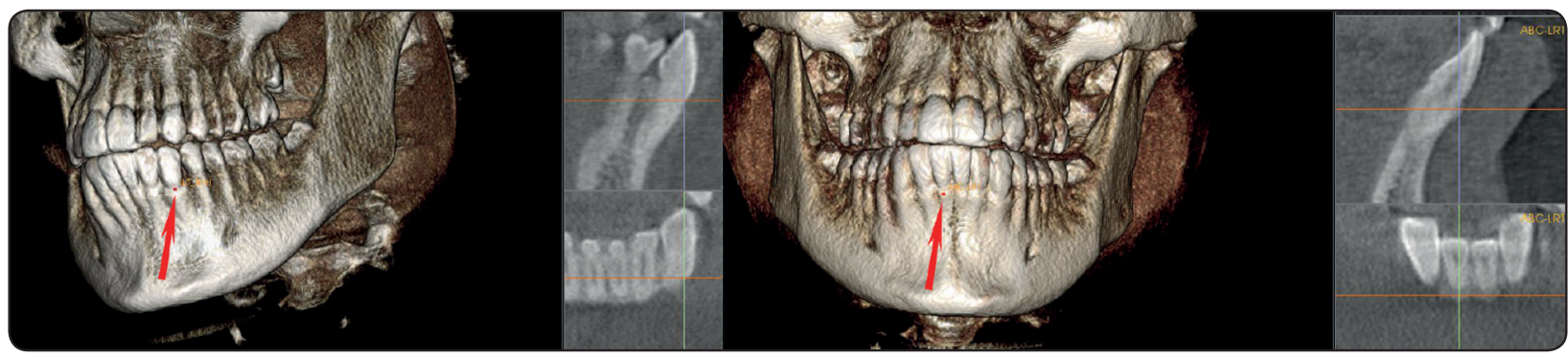

Fig. (1) a \& b: depicting the landmark localization on the CBCT images 


\section{RESULTS}

The results are analyzed for the intra-observer and inter-observer observations. For the intraobserver reliability of the Galileos CBCT scanner, the Intra-Class Coefficient (ICC) tests showed excellent concordance in the $\mathrm{x}, \mathrm{y}$ and $\mathrm{z}$-axes with excellent coefficient in the $95 \%$ confidence interval (Table II). The Mean Absolute Error (MAE) and the Dahlberg Error (DE) showed excellent reproducibility with error $0.38 \mathrm{~mm}$ and $0.37 \mathrm{~mm}$ in the $\mathrm{x}$-direction and $0.53 \mathrm{~mm}$ and $0.55 \mathrm{~mm}$ in the $\mathrm{y}$-direction respectively. However, the error in the $\mathrm{z}$ direction was $0.7 \mathrm{~mm}$ and $0.8 \mathrm{~mm}$ for the MAE and DE respectively. The measurement of intra-observer concordance for the Planmeca CBCT scanner showed excellent concordance using the ICC test. The Mean Absolute Error (MAE) and the Dahlberg Error (DE) showed good reproducibility with error $0.56 \mathrm{~mm}$ and $1.54 \mathrm{~mm}$ in the $\mathrm{x}$-direction and 0.4 $\mathrm{mm}$ and $1.15 \mathrm{~mm}$ in the $\mathrm{y}$-direction respectively. However, the error in the $\mathrm{z}$ direction was $0.56 \mathrm{~mm}$ and $0.76 \mathrm{~mm}$ for the MAE and DE respectively (Table III).

For the inter-observer reliability testing, the same pattern was found for each CBCT scanner, excellent inter-observer reliability for the Galileos scanner in the $\mathrm{x}$, and $\mathrm{y}$ dimensions using the MAE and DE of $0.52 \mathrm{~mm}$ and $0.66 \mathrm{~mm}$ respectively (Table IV), whilst the error in the $\mathrm{z}$ direction was $1 \mathrm{~mm}$ and $1.1 \mathrm{~mm}$ for the MAE and DE respectively. For the inter-observer reliability of the Planmeca CBCT scanner, the Intra-Class Coefficient (ICC) tests showed excellent concordance in the $\mathrm{x}, \mathrm{y}$ and $\mathrm{z}$-axes with excellent coefficient in the $95 \%$ confidence interval. The Mean Absolute Error (MAE) and the Dahlberg Error (DE) showed good reproducibility with error $1.37 \mathrm{~mm}$ and $1.73 \mathrm{~mm}$ in the $\mathrm{x}$-direction, $0.6 \mathrm{~mm}$ and $0.75 \mathrm{~mm}$ in the y-direction respectively. However, the error in the $\mathrm{z}$ direction was $1.2 \mathrm{~mm}$ and $2.4 \mathrm{~mm}$ for the MAE and DE respectively (Table V).

The Dahlberg Error (DE) test uses different formula than the Mean Absolute Error (MAE) for calculating the measurement error. The DE uses a square of the difference, which intensifies and elucidates the error. This test shows high values in cases of difference of large discrepancy even for a few readings. From the displayed results, it is obvious that in some cases, the re-identification of some landmarks by the same observer or by the second observer yielded outlier coordinates, which in turn produces high MAE and DE values (Fig.2\&3).

Independent sample-t-test for comparison between the intra observer landmarks identification between the two scanners showed non-statistically significant difference between the two scanners except in the y-dimension.

Independent sample-t-test for comparison between the inter observer reliability for landmarks identification between the two scanners showed a highly statistically significant difference between the two scanners except in the z-dimension (vertical) which revealed non-statistically significant difference (Table VI). Fig. (4) Depicts the MAE, DE and ICC for comparison of the inter-observer error between the Galileos and the Planmeca CBCT scanners. 
TABLE (II) Showing the intra-observer reliability tests for the Galileos CBCT scanner

\begin{tabular}{|c|c|c|c|c|c|c|c|c|c|}
\hline & & & & & & & \multirow{3}{*}{$\begin{array}{c}\begin{array}{c}\text { Intra- } \\
\text { class }\end{array} \\
\text { ICC } \\
\end{array}$} & \multirow{2}{*}{\multicolumn{2}{|c|}{$\begin{array}{c}\begin{array}{c}\text { Correlation Coef- } \\
\text { ficient }\end{array} \\
\text { 95\% confidence limits }\end{array}$}} \\
\hline & & \multirow[b]{2}{*}{ Mean } & \multirow[b]{2}{*}{ SD } & \multirow[b]{2}{*}{ MAE } & \multirow[b]{2}{*}{ DE } & \multirow[b]{2}{*}{ RDE } & & & \\
\hline & & & & & & & & Lower & Upper \\
\hline \multirow{2}{*}{$\mathbf{X}$} & Measurement 1 & 64.38 & 49.29 & \multirow{2}{*}{1.28} & \multirow{2}{*}{1.22} & \multirow{2}{*}{$1.9 \%$} & \multirow{2}{*}{1.000} & \multirow{2}{*}{1.000} & \multirow{2}{*}{1.000} \\
\hline & Measurement 2 & 64.55 & 49.32 & & & & & & \\
\hline \multirow{2}{*}{$\mathbf{Y}$} & Measurement 1 & 68.67 & 49.13 & \multirow{2}{*}{1.76} & \multirow{2}{*}{1.84} & \multirow{2}{*}{$2.7 \%$} & \multirow{2}{*}{0.999} & \multirow{2}{*}{0.999} & \multirow{2}{*}{1.000} \\
\hline & Measurement 2 & 68.19 & 49.22 & & & & & & \\
\hline \multirow{2}{*}{$\mathbf{Z}$} & Measurement 1 & 98.43 & 54.34 & \multirow{2}{*}{2.37} & \multirow{2}{*}{2.64} & \multirow{2}{*}{$2.7 \%$} & \multirow{2}{*}{0.999} & \multirow{2}{*}{0.998} & \multirow{2}{*}{0.999} \\
\hline & Measurement 2 & 98.70 & 55.10 & & & & & & \\
\hline \multirow{2}{*}{$\mathbf{R}$} & Measurement 1 & 155.43 & 46.25 & \multirow{2}{*}{1.67} & \multirow{2}{*}{1.88} & \multirow{2}{*}{$1.2 \%$} & \multirow{2}{*}{0.999} & \multirow{2}{*}{0.999} & \multirow{2}{*}{0.999} \\
\hline & Measurement 2 & 155.63 & 46.69 & & & & & & \\
\hline
\end{tabular}

MAE: Mean Absolute Error

DE: Dahlberg Error

RDE: Relative Dahlberg Error

TABLE (III) Showing the intra-observer reliability voxel-based tests for the Planmeca CBCT scanner

\begin{tabular}{|c|c|c|c|c|c|c|c|c|c|}
\hline & & & & & & & \multirow{2}{*}{$\begin{array}{l}\text { Intra- } \\
\text { class }\end{array}$} & \multirow{2}{*}{\multicolumn{2}{|c|}{$\begin{array}{c}\begin{array}{c}\text { Correlation } \\
\text { Coefficient }\end{array} \\
\text { 95\%confidence } \\
\text { limits } \\
\end{array}$}} \\
\hline & & & & & & & & & \\
\hline & & Mean & SD & (MAE) & DE & RDE & ICC & Lower & Upper \\
\hline $\mathbf{y}$ & Measurement 1 & 252.12 & 61.14 & 140 & 384 & $15 \%$ & 0008 & 0.008 & 0.008 \\
\hline & Measurement 2 & 252.18 & 61.61 & 1.70 & & 1.07 & 0.770 & 0.790 & 0.770 \\
\hline $\mathbf{Y}$ & Measurement 1 & 127.98 & 59.91 & 007 & 277 & $220 \%$ & 0000 & 0009 & 0.000 \\
\hline & Measurement 2 & 127.87 & 60.03 & & & & & & \\
\hline 7 & Measurement 1 & 251.73 & 53.37 & 136 & 480 & $10 \%$ & 0.096 & 0.005 & 0.007 \\
\hline & Measurement 2 & 250.95 & 52.75 & & & & & & \\
\hline D & Measurement 1 & 383.77 & 78.73 & 127 & & & & 0000 & \\
\hline & Measurement 2 & 383.26 & 78.77 & & & & & & דוניט \\
\hline
\end{tabular}

MAE: Mean Absolute Error

DE: Dahlberg Error

RDE: Relative Dahlberg Error 
TABLE (IV) Showing the inter-observer reliability voxel-based tests for the Galileos CBCT scanner

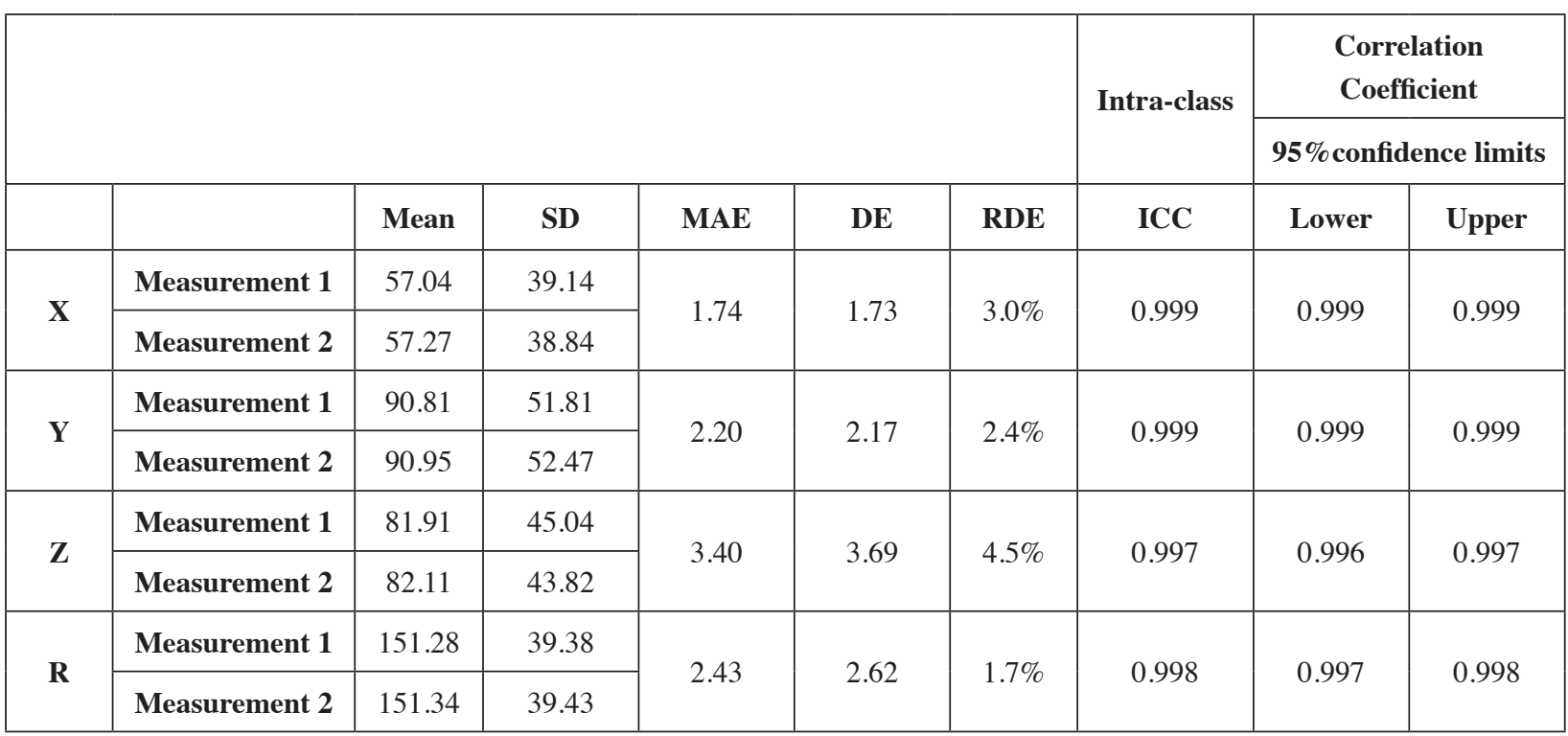

MAE: Mean Absolute Error

DE: Dahlberg Error

RDE: Relative Dahlberg Error

TABLE (V) Showing the inter-observer reliability voxel-based tests for the Planmeca CBCT scanner

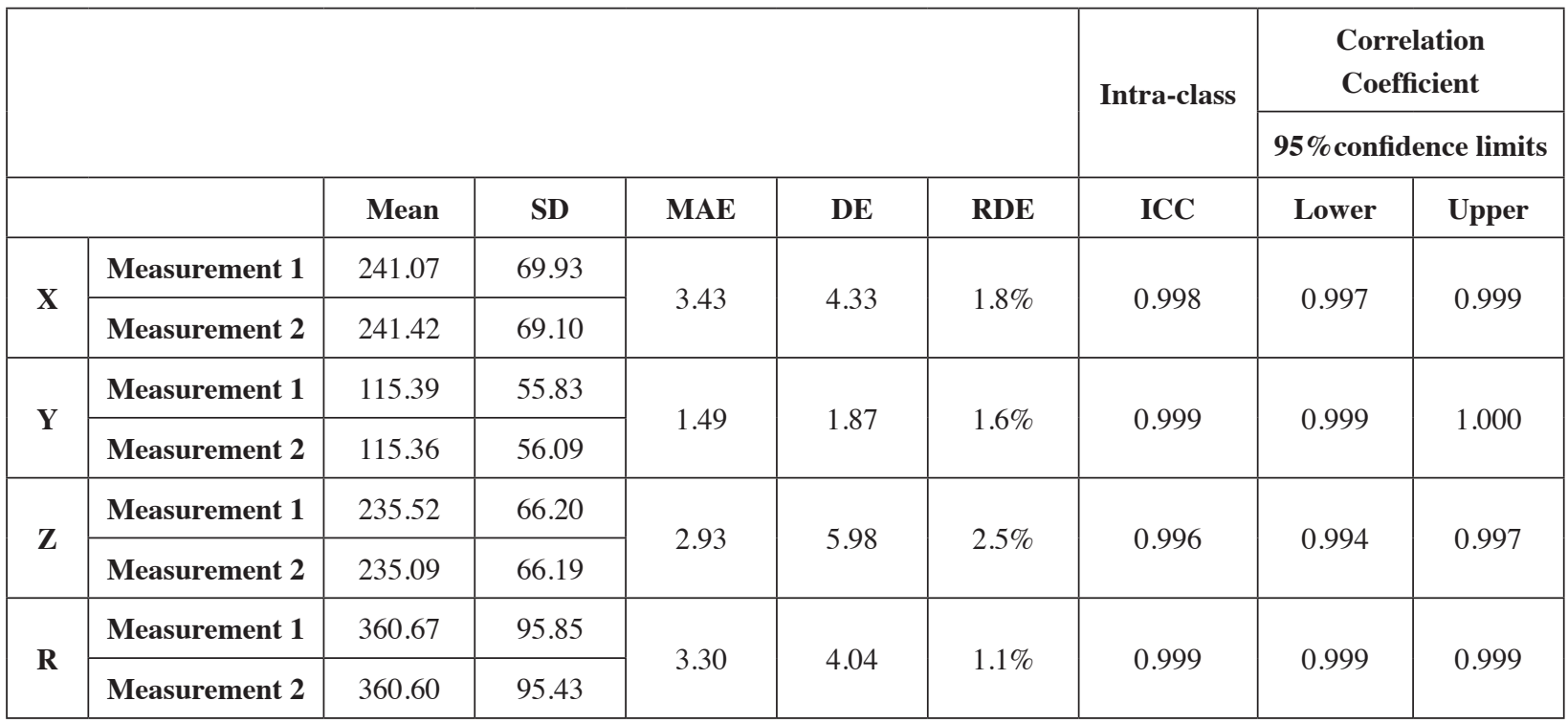

MAE: Mean Absolute Error

DE: Dahlberg Error

RDE: Relative Dahlberg Error 


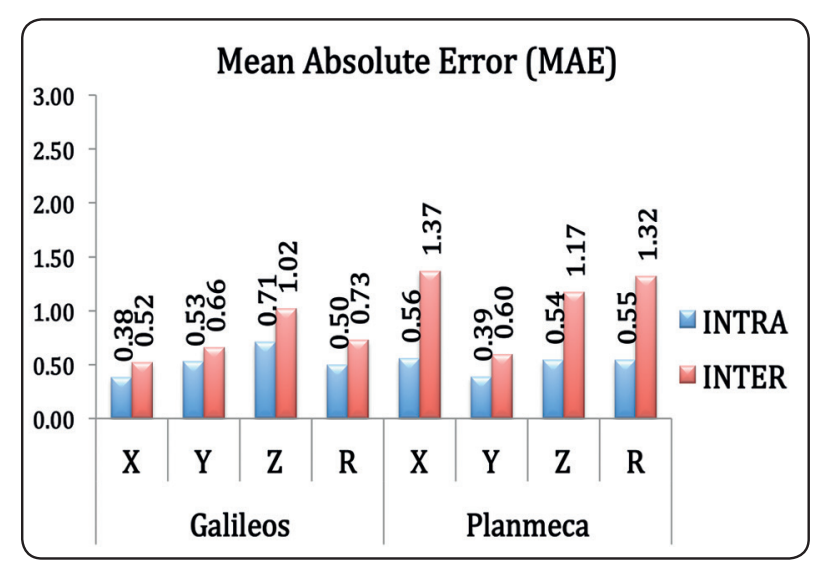

Fig. (2) Shows the Mean Absolute Error in millimeter for the intra-observer and inter-observer reliability for the Galileos and the Planmeca CBCT scanners

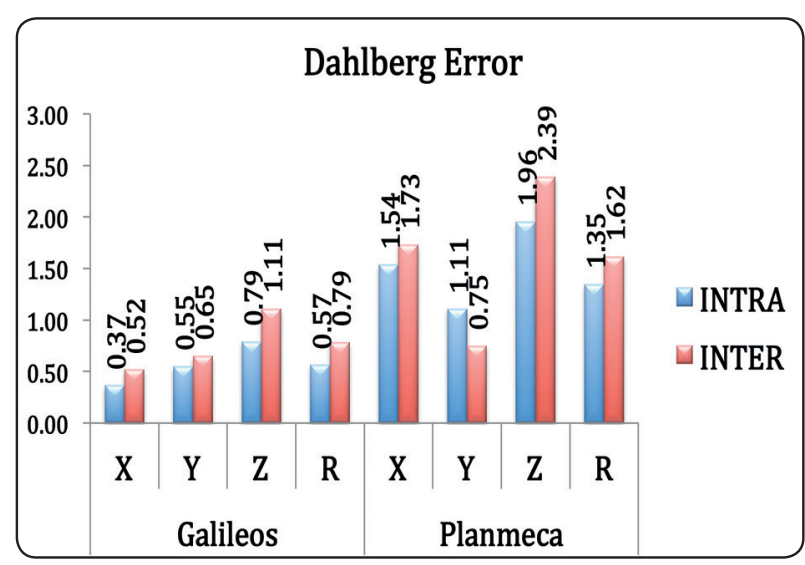

Fig. (3) Shows the Dahlberg Error in millimeter for the intraobserver and inter-observer reliability for the Galileos and the Planmeca CBCT scanners.

TABLE (VI) Independent samples t-test voxel-based for comparing the Intra observer Error between the two machines

\begin{tabular}{|c|c|c|c|c|c|c|c|c|c|c|c|c|}
\hline & & & & & & & & \multicolumn{2}{|c|}{$\begin{array}{c}\text { 95\% Confidence } \\
\text { Interval of the } \\
\text { Difference }\end{array}$} & \multirow[b]{2}{*}{$\mathbf{t}$} & \multirow[b]{2}{*}{ df } & \multirow[b]{2}{*}{ P Value } \\
\hline & & $\mathrm{N}$ & Mean & SD & SEM & $\begin{array}{c}\text { Mean } \\
\text { Difference }\end{array}$ & SED & Lower & Upper & & & \\
\hline \multirow{2}{*}{$\mathbf{X}$} & Galileos & 118 & 1.28 & 1.16 & 0.11 & \multirow{2}{*}{-0.12} & \multirow{2}{*}{0.49} & \multirow{2}{*}{-1.08} & \multirow{2}{*}{0.84} & \multirow{2}{*}{-0.25} & \multirow{2}{*}{468} & \multirow{2}{*}{$0.80279 *$} \\
\hline & Planmeca & 352 & 1.40 & 5.26 & 0.28 & & & & & & & \\
\hline \multirow{2}{*}{$\mathrm{Y}$} & Galileos & 118 & 1.76 & 1.91 & 0.18 & 0.79 & \multirow{2}{*}{0.36} & \multirow{2}{*}{0.07} & \multirow{2}{*}{1.50} & \multirow{2}{*}{2.16} & \multirow{2}{*}{468} & \multirow{2}{*}{$0.03110 *$} \\
\hline & Planmeca & 352 & 0.97 & 3.79 & 0.20 & 0.79 & & & & & & \\
\hline \multirow{2}{*}{$\mathrm{Z}$} & Galileos & 118 & 2.37 & 2.89 & 0.27 & 1.01 & \multirow{2}{*}{0.64} & \multirow{2}{*}{-0.26} & \multirow{2}{*}{2.27} & \multirow{2}{*}{1.56} & \multirow{2}{*}{468} & \multirow{2}{*}{0.11856 * } \\
\hline & Planmeca & 352 & 1.36 & 6.79 & 0.36 & 1.01 & & & & & & \\
\hline \multirow{2}{*}{$\mathrm{R}$} & Galileos & 118 & 1.67 & 2.08 & 0.19 & 0.30 & \multirow{2}{*}{0.43} & \multirow{2}{*}{-0.55} & \multirow{2}{*}{1.16} & \multirow{2}{*}{0.69} & \multirow{2}{*}{468} & \multirow{2}{*}{$0.48953 *$} \\
\hline & Planmeca & 352 & 1.37 & 4.57 & 0.24 & 0.30 & & & & & & \\
\hline
\end{tabular}

$* P>0.05=$ Non-significant difference

**P $<0.05=$ Significant difference 
TABLE (VII) Independent samples t-test voxel-based for comparing the Intra observer Error between the two machines

\begin{tabular}{|c|c|c|c|c|c|c|c|c|c|c|c|c|}
\hline & & & & & & & & \multicolumn{2}{|c|}{$\begin{array}{l}\text { 95\% Confidence } \\
\text { Interval of the } \\
\text { Difference }\end{array}$} & \multirow{2}{*}{\multicolumn{2}{|c|}{ df }} & \multirow[b]{2}{*}{ P Value } \\
\hline & & $\mathrm{N}$ & Mean & SD & SEM & $\begin{array}{c}\text { Mean } \\
\text { Difference }\end{array}$ & SED & Lower & Upper & & & \\
\hline \multirow{2}{*}{$\mathbf{x}$} & Galileos & 280 & 1.74 & 1.72 & 0.10 & \multirow{2}{*}{-1.68} & \multirow{2}{*}{0.33} & \multirow{2}{*}{-2.34} & \multirow{2}{*}{-1.03} & \multirow{2}{*}{-5.06} & \multirow{2}{*}{438} & \multirow{2}{*}{$0.00000 * * *$} \\
\hline & Planmeca & 160 & 3.43 & 5.09 & 0.40 & & & & & & & \\
\hline \multirow{2}{*}{ Y } & Galileos & 280 & 2.20 & 2.14 & 0.13 & 0.71 & \multirow{2}{*}{0.21} & \multirow{2}{*}{0.29} & \multirow{2}{*}{1.13} & \multirow{2}{*}{3.31} & \multirow{2}{*}{438} & \multirow{2}{*}{$0.00102 * * *$} \\
\hline & Planmeca & 160 & 1.49 & 2.19 & 0.17 & 0.71 & & & & & & \\
\hline \multirow{2}{*}{$\mathrm{Z}$} & Galileos & 280 & 3.40 & 3.98 & 0.24 & 0.46 & \multirow{2}{*}{0.57} & \multirow{2}{*}{-0.66} & \multirow{2}{*}{1.58} & \multirow{2}{*}{0.81} & \multirow{2}{*}{438} & \multirow{2}{*}{$0.41552 *$} \\
\hline & Planmeca & 160 & 2.93 & 7.96 & 0.63 & 0.46 & & & & & & \\
\hline \multirow{2}{*}{$\mathrm{R}$} & Galileos & 280 & 2.43 & 2.80 & 0.17 & -0.87 & \multirow{2}{*}{0.36} & \multirow{2}{*}{-1.57} & \multirow{2}{*}{-0.17} & \multirow{2}{*}{-2.45} & \multirow{2}{*}{438} & \multirow{2}{*}{$0.01484 * *$} \\
\hline & Planmeca & 160 & 3.30 & 4.68 & 0.37 & -0.87 & & & & & & \\
\hline
\end{tabular}

*P>0.05=Non-significant difference

$* * * P<0.05=$ Highly significant

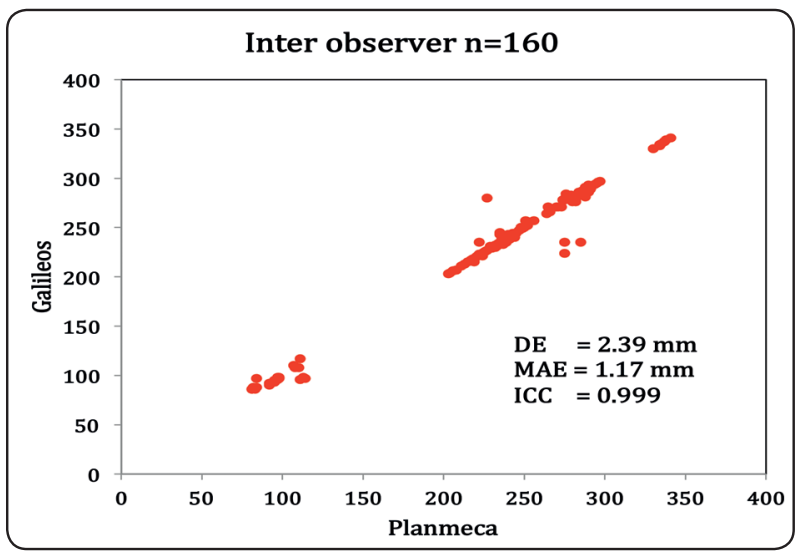

Fig. (4) Depicts the MAE, DE and ICC for comparison of the inter-observer error between the Galileos and the Planmeca CBCT scanners

\section{DISCUSSION}

Undoubtedly, the introduction of CBCT paved new avenues for dentomaxillofacial diagnosis. However, protesters of the ALARA concept still combat against the unjustified use of CBCT. The ALARA policy is a radiation safety principle, which aims at minimizing radiation exposures to individuals. Among the features of the system of dose limitation is the optimization. This means that the radiation exposures resulting from the practice must be reduced to the lowest level possible considering the cost of such a reduction in dose. ${ }^{[32,33]}$

Thus, it seems logical and not to mention ethical, to respect the ALARA and operate the lowest radiation dose $\mathrm{CBCT}$ scanners available in cases 
where CBCT is indicated. The low dose scanner used here within, has been tested for accuracy and 1:1 image-to-reality representation of fiducial markers ${ }^{[34,35]}$. Yet, the low dose produced carries the risk of blending or blurring of fine adjacent structures such as the labial alveolar bone margin opposite the lower incisor teeth in the presence of soft tissue covering in live samples, which provoked their investigation.

The radiation dose rendered by a CBCT scan should be regarded as a function of the diagnostic application required. A key factor for dose optimization, as per the ALARA principles, is to ensure that the scans are performed using the exposure parameters that results in a diagnostically acceptable image for their specific indication.

CBCT systems are rapidly developing towards improving the image quality while keeping the radiation dose as low as possible. CBCT systems vary mainly in their field of view (FOV) and the detector type, with either image intensifier tube and charge coupled device (such as the Galileos scanner) or a flat panel detector (such as the ProMax 3D) [36].

The FOV and accordingly the voxel size are closely related to the spatial resolution of the image ${ }^{[37]}$. The size of voxels plays an integral role in determining the image resolution, quality and scanning times, which has a direct effect on the radiation dose. ${ }^{[38]}$ The radiation dose thus varies between different CBCT depending on FOV and parameters. ${ }^{[39]}$

However, selecting a smaller voxel size, and accordingly a lower radiation dose, carries the risk of a reduced spatial resolution resulting in blending or blurring of fine adjacent structures such as the labial alveolar bone margin opposite the lower incisor teeth in the presence of soft tissue covering in live samples, which provoked this investigation.

Accordingly, this study evaluated the effect of voxel size on the detection of alveolar bone crest at different sites of both dental arches using two different CBCT systems.

The results showed similar intra-observer and inter-observer reliability during landmarks identification in the three axes for the Galileos and the Planmeca scanners. The Galileos scanner with $0.3 \mathrm{~mm}$ voxel size was comparable to the ProMax 3D scanner at a larger voxel size of $0.4 \mathrm{~mm}$. For both scanners, The MAE and the DE values denoted suboptimal reliability in the identification of the alveolar bone crest in the vertical (z) dimension.

It is worth mentioning that both MAE and DE express average model prediction error in units of the variable of interest. Since the errors are squared before they are averaged, the DE gives a relatively high weight to large errors. DE should be more useful when large errors are particularly undesirable.

Similarly, the independent-samples-t-test showed a highly statistically significant difference between the landmarks identification between the two scanners denoting better landmarks identification in the horizontal dimensions ( $\mathrm{x} \& \mathrm{y}$ ), while poor reliability of landmark identification is evident in both scanners in the vertical direction (z).

Sun et al ${ }^{[40]}$ concluded that higher CBCT resolution level $(0.25 \mathrm{~mm}$ vs. $0.4 \mathrm{~mm}$ voxel size) produces more accurate alveolar bone measurements. The smaller the voxel size, the better the resolution should be, and the clearer the image; According to Ballrick et al ${ }^{[30]}, 50 \%$ of the error is attributable to voxel size alone.

However, another factor affecting image quality and resolution is in place, and that is the type of detector. In our case, the Galileos scanner has an IIT/CCD detector while the ProMax 3D scanner has a flat panel detector (Table I). It has been reported that detector type influenced image quality such as IIT/CCD has more artifacts and produces more noise compared with flat panel detector systems ${ }^{[39]}$. However, this study primarily focused on the effect of voxel size on the image quality. 
The greater discrepancy found in the z-coordinate, indicates the challenging localization of the vertical position of the bone margin. Alveolar bone is the junction between cementum and bone, two tissues with similar radio-densities. The labial alveolar bone is considerably thinner in the anterior region and the bone tapers towards the crest, ending into a knife-edge margin opposite the cementum of the teeth.

Besides, when alveolar bone thickness is decreased to a near-pixel level, alveolar bone can become indistinguishable from adjacent structures resulting in inaccuracy of the alveolar bone-height measurements. This incidence was most likely caused by the "invisibility" of the cervical portion of the alveolar crest on CBCT images, a phenomenon which is likely attributable to 2 factors: the inherent limitation of contrast resolution related to CBCT machines. ${ }^{[19,30]}$ and the partial volume averaging effect. ${ }^{[37-39]}$

Contrast resolution is the ability of the CBCT to separate 2 objects of similar densities in close proximity. ${ }^{[40,41]}$ Leung et al ${ }^{[19]}$ using dry human skulls, found that areas with bone less than $0.6 \mathrm{~mm}$ thick were invisible on CBCT images. Anatomically, the alveolar bone is separated from the cementum by the periodontal ligament (approximately $0.5 \mathrm{~mm}$ thick); smaller than this minimum distance requirement, suggesting that the alveolar bone is likely to become indistinguishable from the cementum.

The partial-volume effect is a common CT artifact that occurs when a voxel lies on 2 close objects of different densities. This voxel reflects the average density of both objects rather than the true value of either object. Accordingly, when the alveolar bone thickness is reduced to a level below or near the voxel size, the voxels lying on the alveolar bone will reflect an average density of the alveolar bone and the periodontal ligament, rather than the true value of the alveolar bone. Hence, it becomes indistinguishable from the adjacent periodontal ligament and not considered bone when taking alveolar bone-height measurements. ${ }^{[40]}$

Analysis of the results data indicates that identification of alveolar bone crest on low dose CBCT data is repeatable, reproducible and thus reliable within a margin of error mostly $1 \mathrm{~mm}$. Although there is a non-statistically significant difference in identifying the alveolar bone crest, the amount of error recorded in this study is due to the difficulty to distinguish between very thin bone and the root of the tooth.

The MAE and the DE high values denote poor reliability in the identification of the alveolar bone crest in the vertical dimension. The Galileos machine showed better intra-observer and interobserver reliability during landmarks identification in the three axes than the Planmeca CBCT scanner.

Both MAE and DE express average model prediction error in units of the variable of interest. Since the errors are squared before they are averaged, the DE gives a relatively high weight to large errors. This means the DE should be more useful when large errors are particularly undesirable.

\section{CONCLUSION}

1. The Galileos and the ProMax 3D CBCT scanners produced comparable reliability and reproducibility for identification of alveolar bone crest landmarks.

2. The identification of the alveolar bone crest in the maxillary and mandibular teeth using a low dose CBCT scanner is reliable, ALARA oriented.

3. The vertical axes showed the least accurate coordinates for landmarks identification.

4. Further studies are needed to compare the image-to-reality accuracy of the alveolar bone crest per se using the same CBCT scanners. 


\section{REFERENCES}

1. Kokich, V.G., Cone-beam computed tomography: have we identified the orthodontic benefits? Am J Orthod Dentofacial Orthop, 2010. 137(4 Suppl): p. S16.

2. Carranza F, N.M., Takei H, The tooth-supporting structures. Clinical periodontology. 9th ed. 2002, Philadelphia: W. B. Saunders.

3. Ericsson, I., et al., The effect of orthodontic tilting movements on the periodontal tissues of infected and non-infected dentitions in dogs. J Clin Periodontol, 1977. 4(4): p. 278-93.

4. Wennstrom, J.L., et al., Periodontal tissue response to orthodontic movement of teeth with infrabony pockets. Am J Orthod Dentofacial Orthop, 1993. 103(4): p. 313-9.

5. Artun, J. and K.S. Urbye, The effect of orthodontic treatment on periodontal bone support in patients with advanced loss of marginal periodontium. Am J Orthod Dentofacial Orthop, 1988.93(2): p. 143-8.

6. Rothe, L.E., et al., Trabecular and cortical bone as risk factors for orthodontic relapse. Am J Orthod Dentofacial Orthop, 2006. 130(4): p. 476-84.

7. Thilander, B., Orthodontic relapse versus natural development. Am J Orthod Dentofacial Orthop, 2000. 117(5): p. 562-3.

8. Chaison, J.B., et al., Bone volume, tooth volume, and incisor relapse: a 3-dimensional analysis of orthodontic stability. Am J Orthod Dentofacial Orthop, 2010. 138(6): p. 778-86.

9. Melsen, B. and D. Allais, Factors of importance for the development of dehiscences during labial movement of mandibular incisors: a retrospective study of adult orthodontic patients. Am J Orthod Dentofacial Orthop, 2005. 127(5): p. 552-61; quiz 625.

10. Wennstrom, J.L., Mucogingival considerations in orthodontic treatment. Semin Orthod, 1996. 2(1): p. 46-54.

11. Yared, K.F., E.G. Zenobio, and W. Pacheco, Periodontal status of mandibular central incisors after orthodontic proclination in adults. Am J Orthod Dentofacial Orthop, 2006. 130(1): p. 6 e1-8.

12. Zachrisson, B.U. and L. Alnaes, Periodontal condition in orthodontically treated and untreated individuals. I. Loss of attachment, gingival pocket depth and clinical crown height. Angle Orthod, 1973. 43(4): p. 402-11.
13. Janson, G., et al., Comparative radiographic evaluation of the alveolar bone crest after orthodontic treatment. Am J Orthod Dentofacial Orthop, 2003. 124(2): p. 157-64.

14. Vasconcelos, G., et al., Prevalence and severity of vestibular recession in mandibular incisors after orthodontic treatment. Angle Orthod, 2012. 82(1): p. 42-7.

15. Artun, J. and O. Krogstad, Periodontal status of mandibular incisors following excessive proclination. A study in adults with surgically treated mandibular prognathism. Am J Orthod Dentofacial Orthop, 1987. 91(3): p. 225-32.

16. Steiner, G.G., J.K. Pearson, and J. Ainamo, Changes of the marginal periodontium as a result of labial tooth movement in monkeys. J Periodontol, 1981. 52(6): p. 314-20.

17. Rungcharassaeng, K., et al., Factors affecting buccal bone changes of maxillary posterior teeth after rapid maxillary expansion. Am J Orthod Dentofacial Orthop, 2007. 132(4): p. 428 e1-8.

18. Loubele, M., et al., Comparative localized linear accuracy of small-field cone-beam CT and multislice CT for alveolar bone measurements. Oral Surg Oral Med Oral Pathol Oral Radiol Endod, 2008. 105(4): p. 512-8.

19. Leung, C.C., et al., Accuracy and reliability of conebeam computed tomography for measuring alveolar bone height and detecting bony dehiscences and fenestrations. Am J Orthod Dentofacial Orthop, 2010. 137(4 Suppl): p. S109-19.

20. Evangelista, K., et al., Dehiscence and fenestration in patients with Class I and Class II Division 1 malocclusion assessed with cone-beam computed tomography. Am J Orthod Dentofacial Orthop, 2010. 138(2): p. 133 e1-7; discussion 133-5.

21. Patcas, R., et al., Accuracy of cone-beam computed tomography at different resolutions assessed on the bony covering of the mandibular anterior teeth. Am J Orthod Dentofacial Orthop, 2012. 141(1): p. 41-50.

22. Yagci, A., et al., Dehiscence and fenestration in skeletal Class I, II, and III malocclusions assessed with cone-beam computed tomography. Angle Orthod, 2012. 82(1): p. 67-74.

23. Spin-Neto, R., E. Gotfredsen, and A. Wenzel, Impact of voxel size variation on CBCT-based diagnostic outcome in dentistry: a systematic review. J Digit Imaging, 2013. 26(4): p. 813-20.

24. White, S.C. and M.J. Pharoah, The evolution and application of dental maxillofacial imaging modalities. Dent Clin North Am, 2008. 52(4): p. 689-705, v. 
25. Tsiklakis, K., et al., Dose reduction in maxillofacial imaging using low dose Cone Beam CT. Eur J Radiol, 2005. 56(3): p. 413-7.

26. Grauer, D., L.S. Cevidanes, and W.R. Proffit, Working with DICOM craniofacial images. Am J Orthod Dentofacial Orthop, 2009. 136(3): p. 460-70.

27. Kamburoglu, K., et al., Comparative assessment of subjective image quality of cross-sectional cone-beam computed tomography scans. J Oral Sci, 2011. 53(4): p. 501-8.

28. Katsumata, A., et al., Relationship between density variability and imaging volume size in cone-beam computerized tomographic scanning of the maxillofacial region: an in vitro study. Oral Surg Oral Med Oral Pathol Oral Radiol Endod, 2009. 107(3): p. 420-5.

29. Ludlow, J.B., et al., Dosimetry of 3 CBCT devices for oral and maxillofacial radiology: CB Mercuray, NewTom 3G and i-CAT. Dentomaxillofac Radiol, 2006. 35(4): p. 219-26.

30. Ballrick, J.W., et al., Image distortion and spatial resolution of a commercially available cone-beam computed tomography machine. Am J Orthod Dentofacial Orthop, 2008. 134(4): p. 573-82.

31. Davies, J., B. Johnson, and N. Drage, Effective doses from cone beam CT investigation of the jaws. Dentomaxillofac Radiol, 2012. 41(1): p. 30-6.

32. Health Physics Society. Category: Policy, Guidelines, and Regulations -ALARA.; Available from: http://hps.org.

33. Turpin DL. Clinical guidelines and the use of cone-beam computed tomography. Am J Orthod Dentofacial Orthop
2010. 138: p. 1-2.

34. El-Beialy AR, Fayed MS, El-Bialy AM, Mostafa YA, Accuracy and reliability of cone-beam computed tomography measurements: Influence of head orientation. Am J Orthod Dentofacial Orthop 2011. 140: p. 157-65.

35. Ganguly R, R.A., Vincent S, Hellstein J, Timmons S, Qian $\mathrm{F}$, Accuracy of linear measurement in the Galileos cone beam computed tomography under simulated clinical conditions. Dentomaxillofac Radiol 2011. 40(5): p. 299-305.

36. Alqerban, A., et al., Comparison of 6 cone-beam computed tomography systems for image quality and detection of simulated canine impaction-induced external root resorption in maxillary lateral incisors. Am J Orthod Dentofacial Orthop, 2011. 140(3): p. e129-39.

37. Liang, X., et al., A comparative evaluation of Cone Beam Computed Tomography (CBCT) and Multi-Slice CT (MSCT) Part I. On subjective image quality. Eur J Radiol, 2010. 75(2): p. 265-9.

38. Scarfe, W.C., A.G. Farman, and P. Sukovic, Clinical applications of cone-beam computed tomography in dental practice. J Can Dent Assoc, 2006. 72(1): p. 75-80.

39. Loubele, M., et al., Image quality vs radiation dose of four cone beam computed tomography scanners. Dentomaxillofac Radiol, 2008. 37(6): p. 309-18.

40. Sun, Z., et al., Effect of bone thickness on alveolar boneheight measurements from cone-beam computed tomography images. Am J Orthod Dentofacial Orthop, 2011. 139(2): p. e117-27. 